\title{
Pregnancy Outcomes among Obese Pregnant Women with Varying Levels of Vitamin D in King Abdulaziz University Hospital: A Single-center Retrospective Study
}

Anas M. Fallatah ${ }^{1}$, Areej I. Bahrawi ${ }^{1}$, Hussam Babatin ${ }^{1}$, Khalid M. Nassibi ${ }^{1}$, Yousef AlEdreesi ${ }^{1}$, Hassan Abduljabbar $^{2}$

1. Medicine, King Abdulaziz University, Jeddah, SAU 2. Obstetrics and Gynecology, King Abdulaziz University, Jeddah, SAU

Corresponding author: Anas M. Fallatah, anasmfallatah@gmail.com

\section{Abstract \\ Background}

Vitamin D deficiency among pregnant women is a global issue. Despite its high prevalence, the optimal level of vitamin D among pregnant women is not well established. On the other hand, multiple adverse pregnancy outcomes have been strongly associated with vitamin D deficiency.

\section{Objectives}

To identify the potential effect varying levels of vitamin D have on maternal and neonatal outcomes.

\section{Methods}

This is a non-intervention retrospective record review conducted on pregnant women who delivered in King Abdulaziz University Hospital, Jeddah, Saudi Arabia between January 1, 2013, and December 31, 2018. Data were collected from their hospital electronic files and analyzed by Statistical Package for Social Sciences (SPSS; IBM Corp., Armonk, NY) version 24. A p-value of $<0.05$ is used to calculate statistical significance.

\section{Results}

A total of 9095 pregnant women had delivered in the last five years, 137 of these pregnant women had vitamin D measurement during their pregnancy. Out of 137, 99 were deficient, 20 optimal, 11 therapeutic, and two excess. A total of 97 (70.8\%) were Saudis and 40 (29.2\%) were non-Saudis. The majority of pregnant women in obese class 1 and 2 were deficient with 99 cases, while obese class 3 was mostly those with optimal levels. Regarding pregnancy outcomes, those with vitamin D deficiency had the majority of undesired antepartum, neonatal, and postpartum outcomes. Placenta previa, endometritis, poor APGAR scores, birth

Received 11/19/2019 Review began $11 / 20 / 2019$ Review ended 11/21/2019 Published 11/23/2019

๑) Copyright 2019 Fallatah et al. This is an open access article distributed under the terms of the Creative Commons Attribution License CC-BY 3.0., which permits unrestricted use, distribution, and reproduction in any medium, provided the original author and source are credited. defects, intrauterine fetal demise, low birthweight, and macrosomia were significantly associated with abnormal vitamin D levels $(\mathrm{P}<0.05)$.

\section{Conclusion}

Although vitamin D optimum level during pregnancy is not known, pregnant women with deficient levels appeared to have more serious risks to develop adverse pregnancy outcomes. Therefore, early screening during prenatal visit or antenatal for vitamin D level with vitamin D supplementations is important to reduce these negative pregnancy outcomes for pregnant women with deficient levels.

Categories: Family/General Practice, Obstetrics/Gynecology, Public Health Keywords: vitamin d, maternal, neonatal, pregnancy, obese, bmi, outcomes

\section{Introduction}

Vitamin D is the most micronutrient that has gained and sustained massive interest in the fields of health and biomedical research community [1]. Vitamin D receptors are expressed pervasively in the entire of human body's cells which indicates an involvement of vitamin D-mediated effects on decisive metabolic networks [2]. Vitamin D deficiency is defined as 25(OH) D level to be lower than $50 \mathrm{nmol} / \mathrm{l}(20 \mathrm{ng} / \mathrm{ml})$. In addition, it is an epidemic and it became to be a global issue [3]. Locally, The Middle East and North African (MENA) region has a tremendous prevalence of vitamin D deficiency in comparison to global studies and it is mainly seen in women of varying ages [4]. In Saudi Arabia, according to meta-analysis study made in 2016, it showed that almost $81 \%$ of Saudis are considered to be deficient [1]. Additionally, this pattern is also seen in other Arabian Gulf countries [5-8]. 
Initially, it was believed that deficiency of vitamin D only manifests on bone metabolism, yet now it has become more evident that it has a wide range of extra-skeletal effects such as: neuropsychiatric disorders, deterioration of immune system, increased risk in cardiovascular disease and hypertension, and at last but not least increased risk of mortality [9].

Moreover, race has found to play a major role. African-American adults have been considered to be important risk factor for vitamin D deficiency having the highest prevalence rate of vitamin D deficiency. Other risk factors such as obesity, lack of college education, and lack of daily milk consumption were also identified for vitamin D deficiency [10].

Vitamin D deficiency in pregnant women is common in many parts of the world, and there is a very strong correlation between vitamin D deficiency and multiple adverse pregnancy outcomes [11]. However, the role and metabolism of vitamin D in the pregnancy is not well known [11-14]. In spite of the reported high prevalence of deficiency and the possible consequences, the desired optimal level needed for pregnant women in their body and the amount of vitamin D intake required to maintain adequate levels is not very well documented [11,14-17].

Despite all these studies none of them were done in the western region of Saudi Arabia which encourages us to conduct our research. This study aims to assess the pregnancy outcomes among obese vitamin D deficient pregnant women in King Abdulaziz University Hospital, Jeddah, Saudi Arabia.

\section{Materials And Methods}

This is a retrospective record review study carried out over five years period from January 2013 to December 2018. The study was conducted among obese pregnant women attending King Abdulaziz University Hospital (KAUH), Jeddah, KSA. These electronic medical file records were obtained from the hospital's Medical Records Department. Pregnant women with BMI less than $30 \mathrm{~kg} / \mathrm{m}^{2}$ and missing vitamin D levels were excluded. Out of 1037, 137 patients were found to have vitamin D measurement during their pregnancy and were involved in the study for further analysis. Ethical approval to conduct the study was obtained from KAUH Institutional Review Board with reference number 78-19. Demographic variables such as nationality, gender, age, height, weight, BMI were taken into consideration. Vitamin D metabolites serum concentration was determined by radioimmunoassay kits from Immunodiagnostic Systems Ltd, Boldon, UK. A trained hospital staff (phlebotomist) collected venous blood samples, separated serum, and stored at $-70^{\circ} \mathrm{C}$ until analysis. The treated samples were then assayed using competitive-binding radioimmunoassay. Subjects were classified into five categories: (1) vitamin D deficiency, 25(OH)D <29 ng/mL; (2) adequate, 25(OH)D 30$39 \mathrm{ng} / \mathrm{mL}$; (3) optimal, 25(OH)D 40-59 ng/mL; (4) therapeutic level, between 30 and $80 \mathrm{ng} / \mathrm{mL}$; and (5) excess level, greater than 100 , which is consistent with other studies.

\section{Adverse pregnancy outcomes}

Adverse antepartum variables included the following: Pregnancy-induced hypertension (PTH), preeclampsia, antiphospholipid syndrome (APS), impaired glucose tolerance test (GTT), gestational diabetes mellitus (GDM), pre-premature rupture of membrane (PPROM), premature rupture of membrane (PROM), antepartum hemorrhage (abruptio placenta, placenta previa, placenta accrete and low lying placenta), and maternal mortality; fetomaternal complications (oligohydramnios, polyhydramnios, placental hematoma, and cord prolapse), preterm labor (PTL), induction of labor, mode of delivery (spontaneous vaginal delivery (SVD) or cesarean section (CS)), type of CS (emergency or elective), failure to progress (FTP), cephalopelvic disproportion (CPD), rate of cesarean section, anemia, and urinary tract infection (UTI).

Adverse postpartum variables included the following: Postpartum hemorrhage (PPH), endometritis, vaginal laceration, perianal laceration 1st, 2nd, 3rd degree, internal hemorrhoids, surgical site infection (SSI), and wound dehiscence.

Adverse neonatal outcomes included the following: Gestational age, birth weight, fetal sex, fetal presentation, APGAR score at 1 and 5 minutes, admission to neonatal intensive care unit (NICU), birth defects and injuries, intrauterine fetal demise (IUFD), stillbirth, and neonatal death.

We used the Statistical Package for Social Sciences (SPSS; Release 23.0.0.0, IBM Corp., Armonk, NY). Analysis of data was conducted using SPSS for Windows software. Demographic, antepartum, and postpartum data were examined. The independent $t$-test and correlation tests were used for comparison of quantitative variables. The chi-square test was used as a test of significance for comparison of categorical variables. $\mathrm{P} \leqslant .05$ was chosen as the level of statistical significance.

\section{Results}

The aim of the study is to identify the potential effect varying levels of vitamin D have on maternal and neonatal outcomes. It included 137 pregnant women who delivered their newborn by cesarean section and those which had a BMI measurement of more than 30. Most of the pregnant women were Saudis (97; 70.8\%) while non-Saudis only represented 40 (29.2\%). Table 1 demonstrates the socio-demography of the pregnant 
women. A total of $78.6 \%$ of pregnant women with obese class I had vitamin D deficiency, 22 (16.7\%) of women with obese class II had a deficiency in vitamin D, and none was seen among obese class III, which appeared to be statistically significant with undesired pregnancy outcomes $(\mathrm{P}<0.05)$. Most of vitamin $\mathrm{D}$ deficiencies occurred in pregnant women between the ages of 30 and 34 (29.5\%). Women with multigravida exhibited higher rate of vitamin D deficiency (71.8\%) compared to primigravida. Almost $6.8 \%$ of all preterm neonates had mothers with vitamin D deficiency.

\begin{tabular}{|c|c|c|c|c|c|c|c|c|c|}
\hline & & \multicolumn{8}{|c|}{ Vitamin D levels } \\
\hline & & \multicolumn{2}{|c|}{ Deficient } & \multicolumn{2}{|l|}{ Optimal } & \multicolumn{2}{|c|}{ Therapeutic } & \multicolumn{2}{|l|}{ Excess } \\
\hline & & Count & Table N \% & Count & Table N \% & Count & Table N \% & Count & Table N \% \\
\hline \multirow{2}{*}{ Nationality } & SA & 71 & $53.8 \%$ & 12 & $9.1 \%$ & 9 & $6.8 \%$ & 2 & $1.5 \%$ \\
\hline & $\mathrm{N}-\mathrm{SA}$ & 28 & $21.2 \%$ & 8 & $6.1 \%$ & 2 & $1.5 \%$ & 0 & $0.0 \%$ \\
\hline \multirow{5}{*}{$\mathrm{Age}^{\mathrm{a}}$} & $<30$ & 23 & $17.4 \%$ & 3 & $2.3 \%$ & 1 & $0.8 \%$ & 0 & $0.0 \%$ \\
\hline & $30-34$ & 39 & $29.5 \%$ & 4 & $3.0 \%$ & 4 & $3.0 \%$ & 0 & $0.0 \%$ \\
\hline & $35-39$ & 25 & $18.9 \%$ & 9 & $6.8 \%$ & 4 & $3.0 \%$ & 2 & $1.5 \%$ \\
\hline & $40-44$ & 12 & $9.1 \%$ & 3 & $2.3 \%$ & 2 & $1.5 \%$ & 0 & $0.0 \%$ \\
\hline & $45-50$ & 0 & $0.0 \%$ & 1 & $0.8 \%$ & 0 & $0.0 \%$ & 0 & $0.0 \%$ \\
\hline \multirow{3}{*}{ BMI Group ${ }^{a}$} & Obese Class I & 77 & $58.3 \%$ & 13 & $9.8 \%$ & 7 & $5.3 \%$ & 1 & $0.8 \%$ \\
\hline & Obese Class II & 22 & $16.7 \%$ & 5 & $3.8 \%$ & 4 & $3.0 \%$ & 1 & $0.8 \%$ \\
\hline & Obese Class III & 0 & $0.0 \%$ & 2 & $1.5 \%$ & 0 & $0.0 \%$ & 0 & $0.0 \%$ \\
\hline \multirow{2}{*}{ Gravidity } & Primigravida & 4 & $3.1 \%$ & 3 & $2.3 \%$ & 0 & $0.0 \%$ & 0 & $0.0 \%$ \\
\hline & Multigravida & 94 & $71.8 \%$ & 17 & $13.0 \%$ & 11 & $8.4 \%$ & 2 & $1.5 \%$ \\
\hline \multirow{2}{*}{ Parity } & Nullipara & 5 & $3.8 \%$ & 3 & $2.3 \%$ & 0 & $0.0 \%$ & 0 & $0.0 \%$ \\
\hline & Multipara & 93 & $71.0 \%$ & 17 & $13.0 \%$ & 11 & $8.4 \%$ & 2 & $1.5 \%$ \\
\hline \multirow{3}{*}{ GA } & Preterm & 9 & $6.8 \%$ & 3 & $2.3 \%$ & 2 & $1.5 \%$ & 0 & $0.0 \%$ \\
\hline & Full term & 89 & $67.4 \%$ & 17 & $12.9 \%$ & 9 & $6.8 \%$ & 2 & $1.5 \%$ \\
\hline & Post term & 1 & $0.8 \%$ & 0 & $0.0 \%$ & 0 & $0.0 \%$ & 0 & $0.0 \%$ \\
\hline \multicolumn{10}{|c|}{$\begin{array}{l}\text { TABLE 1: Sociodemographic and maternal characteristics of the studied pregnant women in } \\
\text { relation to vitamin D levels }(n=137)\end{array}$} \\
\hline \multicolumn{10}{|l|}{ a $\mathrm{P}<0.05$} \\
\hline \multicolumn{10}{|c|}{ SA: Saudis; N-SA: Non-Saudis; GA: Gestational age } \\
\hline
\end{tabular}

Maternal and obstetrics characteristics of pregnant woman with complications such as anemia, preeclampsia, and gestational diabetes mellitus are demonstrated in Table 2 . Anemia (71.1\% vs $28.9 \%$ ) and GDM (54.2\% vs 45.8\%) was more frequent in Saudi pregnant women than in non-Saudi pregnant woman. One-third of women between the ages of 35 and 39 developed gestational diabetes mellitus. Anemia occurred most frequently to woman between the ages of 30 and 34 (37.8\%). Furthermore, only two patients who suffered severe preeclampsia were Saudi, and two out of the three patients who developed mild preeclampsia were non-Saudi. Urinary tract infections in Saudi women were higher than in non-Saudi woman during pregnancy. Also, patients with obese class I had higher rates of UTIs (68.3\%), GDM (66.7\%), and anemia (70\%) compared to other groups. Multigravida pregnant women exhibited higher rates of anemia (94.4\%), GDM (95.8\%), and UTIs (92.7\%) compared to primigravida. More than half of the females with GDM underwent an elective cesarean section. 


\section{Cureus}

\begin{tabular}{|c|c|c|c|c|c|c|c|c|c|c|c|}
\hline \multirow{3}{*}{ Nationality } & \multirow[b]{2}{*}{ SA } & \multicolumn{2}{|c|}{ Mild Preeclampsia } & \multicolumn{2}{|c|}{ Severe Preeclampsia } & \multicolumn{2}{|c|}{ Anemia } & \multicolumn{2}{|c|}{ GDM } & \multicolumn{2}{|c|}{ UTIs } \\
\hline & & 1 & $33.3 \%$ & 2 & $100.0 \%$ & 64 & $71.1 \%$ & 13 & $54.2 \%$ & 31 & $75.6 \%$ \\
\hline & $\mathrm{N}-\mathrm{SA}$ & 2 & $66.7 \%$ & 0 & $0.0 \%$ & 26 & $28.9 \%$ & 11 & $45.8 \%$ & 10 & $24.4 \%$ \\
\hline \multirow{3}{*}{$\mathrm{BMI} \mathrm{B}^{\mathrm{a}}$} & Obese Class I & 1 & $33.3 \%$ & 0 & $0.0 \%$ & 63 & $70.0 \%$ & 16 & $66.7 \%$ & 28 & $68.3 \%$ \\
\hline & Obese Class II & 2 & $66.7 \%$ & 2 & $100.0 \%$ & 25 & $27.8 \%$ & 8 & $33.3 \%$ & 12 & $29.3 \%$ \\
\hline & Obese Class III & 0 & $0.0 \%$ & 0 & $0.0 \%$ & 2 & $2.2 \%$ & 0 & $0.0 \%$ & 1 & $2.4 \%$ \\
\hline \multirow{5}{*}{ Age } & $<30$ & 1 & $33.3 \%$ & 0 & $0.0 \%$ & 14 & $15.6 \%$ & 4 & $16.7 \%$ & 10 & $24.4 \%$ \\
\hline & $30-34$ & 1 & $33.3 \%$ & 0 & $0.0 \%$ & 34 & $37.8 \%$ & 5 & $20.8 \%$ & 13 & $31.7 \%$ \\
\hline & $35-39$ & 1 & $33.3 \%$ & 1 & $50.0 \%$ & 28 & $31.1 \%$ & 8 & $33.3 \%$ & 10 & $24.4 \%$ \\
\hline & $40-44$ & 0 & $0.0 \%$ & 1 & $50.0 \%$ & 14 & $15.6 \%$ & 7 & $29.2 \%$ & 8 & $19.5 \%$ \\
\hline & $45-50$ & 0 & $0.0 \%$ & 0 & $0.0 \%$ & 0 & $0.0 \%$ & 0 & $0.0 \%$ & 0 & $0.0 \%$ \\
\hline \multirow{2}{*}{ Parity } & Nulliparous & 0 & $0.0 \%$ & 0 & $0.0 \%$ & 5 & $5.6 \%$ & 1 & $4.2 \%$ & 3 & $7.3 \%$ \\
\hline & Multipara & 3 & $100.0 \%$ & 2 & $100.0 \%$ & 85 & $94.4 \%$ & 23 & $95.8 \%$ & 38 & $92.7 \%$ \\
\hline \multirow{2}{*}{ Gravidit } & Prim gravida & 0 & $0.0 \%$ & 0 & $0.0 \%$ & 5 & $5.6 \%$ & 1 & $4.2 \%$ & 3 & $7.3 \%$ \\
\hline & Multigravida & 3 & $100.0 \%$ & 2 & $100.0 \%$ & 85 & $94.4 \%$ & 23 & $95.8 \%$ & 38 & $92.7 \%$ \\
\hline
\end{tabular}

TABLE 2: Maternal and obstetrics characteristics with complications such as anemia, preeclampsia, gestational diabetes mellitus (GDM), and urinary tract infections ( $\mathrm{n}=137$ )

a $\mathrm{P}<0.05$

GDM: Gestational diabetes mellitus; UTIs: Urinary tract infections; SA: Saudis; N-SA: Non-Saudis.

Table 3 shows the vitamin D levels in pregnant women with maternal morbidity. Vitamin D deficiency was higher in pregnant women with anemia (49.2\%). However, vitamin D deficiency was found in only $12.1 \%$ females with GDM (3\%) with mild preeclampsia, and $2 \%$ with severe preeclampsia. While the most prevalent placental pathology among our sample was placenta previa, which was more commonly seen among pregnant women with deficient vitamin $\mathrm{D}$, which showed a significant association $(\mathrm{P}<0.05)$. One case of PTL was also seen in a pregnant woman with vitamin D deficiency. Moreover, there was also one case seen in a pregnant woman with therapeutic levels of vitamin $\mathrm{D}$, which showed significant association $(\mathrm{P}<0.05)$. A total of 11 cases had induction to terminate their pregnancy, most of them were also vitamin D deficient. Surprisingly, SSI appeared to be seen only in pregnant women with deficient vitamin D levels. Regarding UTI, it was also highly seen among pregnant women with vitamin D deficiency, with 31 cases. 


\section{Cureus}

\begin{tabular}{|c|c|c|c|c|}
\hline & \multicolumn{4}{|l|}{ Vitamin D Levels } \\
\hline & Deficient $(\mathrm{n}=99)$ & Optimal $(n=20)$ & Therapeutic $(n=11)$ & Excess $(n=2)$ \\
\hline Mild Preeclampsia & $3(3 \%)$ & 0 & 0 & 0 \\
\hline Severe Preeclampsia & $2(2 \%)$ & 0 & 0 & 0 \\
\hline GDM & $16(12.1 \%)$ & $3(15 \%)$ & $3(27.3 \%)$ & $1(50 \%)$ \\
\hline PIH & $1(1 \%)$ & $2(10 \%)$ & 0 & 0 \\
\hline PROM & 0 & 0 & 0 & 0 \\
\hline Placenta Previa ${ }^{a}$ & $4(4 \%)$ & 0 & 0 & 0 \\
\hline Abruptio Placenta & $1(1 \%)$ & 0 & 0 & 0 \\
\hline Low Lying Placenta & 0 & 0 & 0 & 0 \\
\hline Placenta Accreta & $1(1 \%)$ & 0 & 0 & 0 \\
\hline Maternal Mortality & 0 & 0 & 0 & 0 \\
\hline Preterm Labor & $1(1 \%)$ & 0 & 0 & 0 \\
\hline Induction of Labor & $7(7.1 \%)$ & $2(10 \%)$ & 1 & 1 \\
\hline PPH & 0 & 0 & 0 & 0 \\
\hline Endometritis $^{\mathrm{a}}$ & 0 & 0 & 1 & 0 \\
\hline SSI & $3(3 \%)$ & 0 & 0 & 0 \\
\hline Anemia & $65(65.7 \%)$ & $12(60 \%)$ & $8(72.7 \%)$ & 2 (100\%) \\
\hline UTI & $31(31.3 \%)$ & $4(20 \%)$ & $3(27.3 \%)$ & $1(50 \%)$ \\
\hline
\end{tabular}

TABLE 3: Vitamin D levels in pregnant women with different maternal complications

a $P<0.05$

GDM: Gestational diabetes mellitus; PIH: Pregnancy-induced hypertension; PROM: Premature rupture of membranes; PPH: Postpartum hemorrhage; SSI: Surgical site infection; UTI: Urinary tract infection.

Table 4 displays frequency of maternal comorbidities. In the observed sample, $19.7 \%$ of the studied pregnant women have comorbidities, of those $14.4 \%$ had vitamin D deficiency. Hypothyroidism was the most prevalent comorbidity in the sample, representing $8 \%$ of all comorbidities. Hypertension (HTN) was the second most common comorbidity representing only 4.4\%. While diabetes mellitus type 1 and type 2 had been represented with three and two cases, respectively. 


\section{Cureus}

\begin{tabular}{|c|c|c|c|c|}
\hline & \multicolumn{4}{|c|}{ Vitamin D Levels } \\
\hline & Deficient & Optimal & Therapeutic & Excess \\
\hline Maternal Comorbidity & 22 & 4 & 4 & 1 \\
\hline HTN & $5(22.7 \%)$ & 0 & $1(25 \%)$ & 0 \\
\hline DM Type 1 & $2(9.1 \%)$ & 0 & 0 & 0 \\
\hline DM Type 2 & $3(13.6 \%)$ & 0 & $1(25 \%)$ & 0 \\
\hline SLE & $1(4.5 \%)$ & 0 & 0 & 0 \\
\hline Hypothyroidism & $6(27.2 \%)$ & $3(75 \%)$ & $1(25 \%)$ & $1(100 \%)$ \\
\hline Neurological Disease & $1(4.5 \%)$ & 0 & 0 & 0 \\
\hline Psychiatric illness & $2(9.1 \%)$ & 0 & 0 & 0 \\
\hline Bronchial Asthma & $1(4.5 \%)$ & 0 & $1(25 \%)$ & 0 \\
\hline Hyperlipidemia & 0 & $1(25 \%)$ & 0 & 0 \\
\hline Rheumatic Heart Disease & $1(4.5 \%)$ & 0 & 0 & 0 \\
\hline
\end{tabular}

TABLE 4: Frequency of morbidity among the studied pregnant females with varying vitamin $D$ levels $(n=137)$

HTN: Hypertension; DM: Diabetes mellitus; SLE: Systemic lupus erythematosus.

Regrading neonatal outcomes from Table 5, most of the neonates who had low birthweight born to mothers with a deficient vitamin D level in comparison to optimal, therapeutic and excess levels. Yet, more than half of neonates born to vitamin D deficient mothers had normal birth weight. Both of these findings had significant association with p-value less than 0.001. On the other hand, poor Apgar scores at 1 minute were higher in women with vitamin D deficiency (13 cases), while those with optimal and therapeutic levels had less frequent events with 2 and 3, respectively. These results were also statistically significant with p-value less than 0.001 . In addition, $8 \%$ of neonates were admitted to newborn intensive care unit (NICU) and most of them were related to mothers with deficient vitamin D levels. One case of IUFD was found in a pregnant woman with therapeutic levels of vitamin $\mathrm{D}$, which is also statistically significant with p-value less than 0.001. Surprisingly, there is no event of oligohydramnios or polyhydramnios in our sample. 


\section{Cureus}

\begin{tabular}{|c|c|c|c|c|c|}
\hline & & \multicolumn{4}{|c|}{ Vitamin D Levels } \\
\hline & & Deficient & Optimal & Therapeutic & Excess \\
\hline \multirow{6}{*}{ Birthweight $^{b}$} & \multirow{2}{*}{ Low Birthweight } & 11 & 3 & 1 & 0 \\
\hline & & $11.2 \%$ & $16.7 \%$ & $9.1 \%$ & $0.0 \%$ \\
\hline & \multirow{2}{*}{ Normal Birthweight } & 84 & 14 & 10 & 2 \\
\hline & & $85.7 \%$ & $77.8 \%$ & $90.9 \%$ & $100 \%$ \\
\hline & \multirow{2}{*}{ Macrosomia } & 3 & 1 & 0 & 0 \\
\hline & & $3.1 \%$ & $5.6 \%$ & $0.0 \%$ & $0.0 \%$ \\
\hline Poor APGAR at $1 \mathrm{~min}^{\mathrm{b}}$ & & $13(13.3 \%)$ & $2(11.1 \%)$ & $3(27.3 \%)$ & 0 \\
\hline Poor APGAR at $5 \mathrm{~min}^{\mathrm{b}}$ & & 0 & 0 & 0 & 0 \\
\hline Birth Defects ${ }^{a}$ & & $2(2 \%)$ & 0 & $1(9.1 \%)$ & 0 \\
\hline IUFD $^{a}$ & & 0 & 0 & $1(9.1 \%)$ & 0 \\
\hline NICU Admission & & $8(8.1 \%)$ & $2(10 \%)$ & $1(9.1 \%)$ & 0 \\
\hline Oligohydramnios & & 0 & 0 & 0 & 0 \\
\hline Polyhydramnios & & 0 & 0 & 0 & 0 \\
\hline
\end{tabular}

TABLE 5: Frequency of adverse neonatal outcomes among our sample $(n=137)$

a $P<0.05$

${ }^{b} \mathrm{P}<0.05$

IUFD: Intrauterine fetal demise; NICU: Neonatal intensive care unit.

From Table 6, we can see the most frequent fetal presentation is cephalic (82.3\%) unlike breech which was only $12.4 \%$. Most fetuses in the study were female (51.8\%) with male estimated at $48.2 \%$.

\begin{tabular}{|c|c|c|c|}
\hline & & Count & Percentage \\
\hline \multirow{2}{*}{ Fetal Sex } & Male & 66 & $48.2 \%$ \\
\hline & Female & 71 & $51.8 \%$ \\
\hline \multirow{2}{*}{ Fetal Presentation } & Cephalic & 79 & $82.3 \%$ \\
\hline & Breech & 17 & $17.7 \%$ \\
\hline
\end{tabular}

TABLE 6: Frequency of fetal characteristics

\section{Discussion}

The findings of this study have displayed the widespread presence of vitamin D deficiency among obese pregnant women in KAUH, Jeddah. From our study, its prevalence was most marked in those who are obese class 1 , whereas a lower prevalence was found in those who are obese class 2 . A study conducted in Saudi Arabia and directed towards vitamin D deficiency among pregnant Saudi women displayed similar results [18]. Most of the study participants were either overweight or obese with the majority of pregnant women (91.9\%) having vitamin D deficiency. On the contrary, a study conducted in Southern China showed the prevalence of vitamin D deficiency to be at $18.9 \%$, with no significant differences in most adverse pregnancy outcomes [19].

A systematic review and meta-analysis confirmed the association between obesity and vitamin D deficiency 
within different age groups [20]. They also showed higher prevalence of vitamin D deficiency was $35 \%$ higher in obese subjects compared to non-obese subjects. Although, the study's focus was not directed towards pregnant women, the clear effect of obesity on vitamin D deficiency was noted. Another recent study conducted in Spain, reported vitamin D deficiency in a high percentage of obese and overweight northern Spanish pregnant women (34.5\%) [21]. It can be observed that the previously mentioned studies have had similar findings to ours.

According to a recent study conducted in India, complications including gestational diabetes, preeclampsia, and preterm labor were associated with vitamin D deficiency in pregnant women, which supports our study result that has also highlighted the complications at risk in pregnant obese women with different vitamin D levels [22]. Complications included anemia, gestational diabetes mellitus, preeclampsia, and urinary tract infections to name a few. On the other hand, those with excess levels of vitamin D were associated with GDM, and preterm labor according to a recent study [19]. These complications came to support our results, which showed pregnant women with excess levels of vitamin D developed GDM, induction of labor, anemia, and UTI.

The aforementioned study also displayed low birth weight and poor Apgar score in the neonates born to the mothers, which we also found regarding neonates of vitamin D deficient mothers that displayed a number of complications including low birth weight, macrosomia, poor APGAR scores at $1 \mathrm{~min}$, and admission to the NICU although more than half had normal birthweight [19].

Another study, we also took note of, was a study conducted on the effects of vitamin D deficiency in pregnancy which states that effects of vitamin D may occur earlier in pregnancy than previously appreciated. And that to date, most studies regarding this topic focus on vitamin D deficiency in later stages of pregnancy [23].

A study conducted in the USA showed pregnant women with optimal levels of vitamin D were protected against adverse pregnancy outcomes with $57 \%$ lower risk than those with vitamin D deficiency [24]. Yet, our results showed those pregnant women with optimal levels of vitamin D developed GDM, pregnancy-induced hypertension (PIH), induction of labor, anemia, and UTI. Not only that, they also had low birth weight, macrosomia, poor APGAR scores, and NICU admission rates. Although these results were only seen in small portion of those with optimal vitamin D levels in comparison to vitamin D deficient but the relation remains unclear.

As for pregnant women with therapeutic levels of vitamin D, similar complications of those with optimal and deficient levels of vitamin D were present with the exception of PIH. All except for GDM were displayed in lower rates when compared to pregnant women with deficient and optimal levels of vitamin D. Regarding the neonates, low birth weight and NICU admission were found but similarly in low rates. Poor Apgar at 1 min was higher than in neonates born to mothers with optimal levels of vitamin D. Compared to a recent systemic review conducted from 13 randomized controlled trials (RCTs) it showed that incidences of preeclampsia, GDM, SGA, low birth weight, preterm birth, and cesarean section were not influenced by vitamin D status [25].

The WHO states that vitamin D supplementation improves maternal vitamin D status and may reduce the risk of pre-eclampsia, low birth weight and preterm birth. However, the data regarding the direct benefits and harms of supplementation is fairly limited. Therefore, the WHO does not recommend vitamin D supplementation to improve maternal and perinatal outcomes. This supersedes the previous recommendation in 2012 [25]. Although a study from the American Society for Bone and Mineral Research addresses that there was no harm from implementing vitamin D supplementation or screening for all pregnant women [26].

The following is enlisted in the American College of Obstetricians and Gynecologists: At this time there is insufficient evidence to support a recommendation for screening all pregnant women for vitamin D deficiency. However, we may consider screening for pregnant women thought to be at risk. $25(\mathrm{OH}) \mathrm{D}$ is used for screening and should be interpreted in the context of the individual circumstance. Most experts would agree that supplementing a 1000 to 2500 international units is safe. Higher dose regimens during pregnancy have not yet been studied [27]. In conclusion, vitamin D status seems to contribute to pregnancy outcome on both the maternal and neonatal level.

As what is usually the case, the study could have benefited from a larger sample. But then again as the title implies it is a single center study and hopefully other local centers could make use of the data if they wish to conduct a similar study. Also, in contrast to other global studies, our sample is probably more prone to vitamin D deficiency owing to the relatively less sun exposure, which is also observed in studies in other countries with similar lifestyle such as Iran and other Middle East countries, which in turn would lead to a higher prevalence of vitamin D deficiency possibly having an impact on the results. A similar relationship can be observed but in an inversely manner in the study from Southern China, which demonstrated a much lower prevalence and no significant difference in outcome. 


\section{Conclusions}

As our study showed, obese pregnant women with vitamin D deficiency are more prone to adverse maternal and neonatal outcomes. Due to the high prevalence of vitamin D deficiency in our region, an early screening during preconception period or first antenatal visit is vital. As we mentioned earlier, those who are deficient are going to benefit from vitamin D supplementation. At the end, we again recommend a population-based multi-center cohort study to provide more accurate knowledge about prevalence and impact not only of vitamin D deficiency but also other varying levels on pregnancy outcomes.

\section{Appendices}

Author's contributions:

Each author gave substantial contribution to the conception or design of the work and in the acquisition, analysis and interpretation of data for the work. Each author had role in drafting the work and revising it critically. Each author gave final approval of the version to be published and they agree to be accountable for all aspects of the work in ensuring that questions related to the accuracy or integrity of any part of the work are appropriately investigated and resolved.

Conflict of Interest: None.

\section{Additional Information \\ Disclosures}

Human subjects: Consent was obtained by all participants in this study. Animal subjects: All authors have confirmed that this study did not involve animal subjects or tissue. Conflicts of interest: In compliance with the ICMJE uniform disclosure form, all authors declare the following: Payment/services info: All authors have declared that no financial support was received from any organization for the submitted work. Financial relationships: All authors have declared that they have no financial relationships at present or within the previous three years with any organizations that might have an interest in the submitted work. Other relationships: All authors have declared that there are no other relationships or activities that could appear to have influenced the submitted work.

\section{References}

1. Al-Daghri NM: Vitamin D in Saudi Arabia: prevalence, distribution and disease associations . J Steroid Biochem Mol Biol. 2018, 175:102-107. 10.1016/j.jsbmb.2016.12.017

2. Prasad P, Kochhar A: Interplay of vitamin D and metabolic syndrome: a review . Diabetes Metab Syndr. 2016, 10:105-112. 10.1016/j.dsx.2015.02.014

3. Holick MF, Chen TC: Vitamin D deficiency: a world wide problem with health consequences . Am J Clin Nutr. 2008, 87:1080-1086. 10.1093/ajcn/87.4.1080S

4. Maalouf G, Gannage-Yared MH, Ezzedine J, et al.: Middle East and North Africa consensus on osteoporosis . J Muskuloskelet Neuronal Interact. 2007, 7:131-143.

5. Haq A, Svobodová J, Imran S, Stanford C, Razzaque MS: Vitamin D deficiency: a single centre analysis of patients from 136 countries. J Steroid Biochem Mol Biol. 2016, 164:209-213. 10.1016/j.jsbmb.2016.02.007

6. Zhang FF, Al Hooti S, Al Zenki S, et al.: Vitamin D deficiency is associated with high prevalence of diabetes in Kuwaiti adults: results from a national survey. BMC Public Health. 2016, 16:100. 10.1186/s12889-0162758-x

7. Golbahar J, Al-Saffar N, Diab DA, Al-Othman S, Darwish A, Al-Kafaji G: Predictors of vitamin D deficiency and insufficiency in adult Bahrainis: a cross-sectional study. Public Health Nutr. 2014, 17:732-738. 10.1017/S136898001300030X

8. Gerber LM, Giambrone AE, Al-Ali HM, Verjee MA: Validity of self-reported vitamin D deficiency among midlife Arab women living in Qatar. Am J Hum Biol. 2016, 28:181-185. 10.1002/ajhb.22766

9. Balion C, Griffith LE, Strifler L, et al.: Vitamin D, cognition, and dementia: a systematic review and metaanalysis. Neurology. 2012, 79:1397-1405. 10.1212/WNL.0b013e31826c197f

10. Forrest KY, Stuhldreher WL: Prevalence and correlates of vitamin D deficiency in US adults . Nutr Res. 2011, 31:48-54. 10.1016/j.nutres.2010.12.001

11. Wagner CL, Taylor SN, Dawodu A, Johnson DD, Hollis BW: Vitamin D and its role during pregnancy in attaining optimal health of mother and fetus. Nutrients. 2012, 4:208-230. 10.3390/nu4030208

12. Holick MF, Binkley NC, Bischoff-Ferrari HA, et al.: Evaluation, treatment, and prevention of vitamin D deficiency: an Endocrine Society clinical practice guideline. J Clin Endocrinol Metab. 2011, 96:1911-1930. 10.1210/jc.2011-0385

13. Javaid MK, Crozier SR, Harvey NC, et al.: Maternal vitamin D status during pregnancy and childhood bone mass at age 9 years: a longitudinal study. Lancet. 2006, 367:36-43. 10.1016/S0140-6736(06)67922-1

14. Bowyer L, Catling-Paull C, Diamond T, Homer C, Davis G, Craig ME: Vitamin D, PTH and calcium levels in pregnant women and their neonates. Clin Endocrinol. 2009, 70:372-377. 10.1111/j.1365-2265.2008.03316.x

15. Dawodu A, Akinbi H: Vitamin D nutrition in pregnancy: current opinion . Int J Women's Health. 2013, 5:333343. 10.2147\%2FIJWH.S34032

16. Hamilton SA, McNeil R, Hollis BW, et al.: Profound vitamin D deficiency in a diverse group of women during pregnancy living in a sun-rich environment at latitude $32^{\circ} \mathrm{N}$. Int J Endocrinol. 2010, 2010:10. $10.1155 / 2010 / 917428$ 
17. Sahu M, Bhatia V, Aggarwal A, Rawat V, Saxena P, Pandey A, Das V: Vitamin D deficiency in rural girls and pregnant women despite abundant sunshine in northern India. Clin Endocrinol. 2009, 70:680-684. 10.1111/j.1365-2265.2008.03360.x

18. Al-Faris N: High prevalence of vitamin D deficiency among pregnant Saudi women . Nutrients. 2016, 8:77. 10.3390/nu8020077

19. Zhou J, Su L, Liu M, Liu Y, Cao X, Wang Z, Xiao H: Associations between 25-hydroxyvitamin D levels and pregnancy outcomes: a prospective observational study in southern China. Eur J Clin Nutr. 2014, 68:925930. 10.1038/ejcn.2014.99

20. Pereira-Santos M, Costa PR, Assis AM, Santos CA, Santos DB: Obesity and vitamin D deficiency: a systematic review and meta-analysis. Obes Rev. 2015, 16:341-349. 10.1111/obr.12239

21. Rodríguez-Dehli AC, Riaño IG, Fernandez-Somoano A, Navarrete-Muñoz EM, Espada M, Vioque J, Tardon A: Prevalence of vitamin D deficiency and insuffiency and associated factors in pregnant women of northern Spain. Nutr Hosp. 2015, 31:1633-1640. 10.3305/nh.2015.31.4.8448

22. Sablok A, Batra A, Thariani K, et al.: Supplementation of vitamin D in pregnancy and its correlation with feto-maternal outcome. Clin Endocrinol. 2015, 83:536-541. 10.1111/cen.12751

23. Ganguly A, Tamblyn JA, Finn-Sell S, et al.: Vitamin D, the placenta and early pregnancy: effects on trophoblast function. J Endocrinol. 2018, 236:93-103. 10.1530/JOE-17-0491

24. Wagner CL, Baggerly C, McDonnell S, et al.: Post-hoc analysis of vitamin D status and reduced risk of preterm birth in two vitamin D pregnancy cohorts compared with South Carolina March of Dimes 2009-2011 rates. J Steroid Biochem Mol Biol. 2016, 155:245-251. 10.1016/j.jsbmb.2015.10.022

25. Vitamin D supplementation during pregnancy. (2019). Accessed: September 29, 2019: https://www.who.int/elena/titles/vitamind_supp_pregnancy/en/.

26. Hollis BW, Johnson D, Hulsey TC, Ebeling M, Wagner CL: Vitamin D supplementation during pregnancy: double-blind, randomized clinical trial of safety and effectiveness. J Bone Miner Res. 2011, 26:2341-2357. 10.1002/jbmr.463

27. ACOG Committee on Obstetric Practice: ACOG Committee Opinion No. 495: Vitamin D: screening and supplementation during pregnancy. Obstet Gynecol. 2011, 118:197-198. 10.1097/AOG.0b013e318227f06b 